\title{
Research Article \\ Effects of Concrete on Propagation Characteristics of Guided Wave in Steel Bar Embedded in Concrete
}

\author{
Zhupeng Zheng and Ying Lei \\ Department of Civil Engineering, Xiamen University, Xiamen 361005, China \\ Correspondence should be addressed to Ying Lei; ylei@xmu.edu.cn
}

Received 22 June 2014; Revised 22 July 2014; Accepted 28 July 2014; Published 27 August 2014

Academic Editor: Ting-Hua Yi

Copyright (C) 2014 Z. Zheng and Y. Lei. This is an open access article distributed under the Creative Commons Attribution License, which permits unrestricted use, distribution, and reproduction in any medium, provided the original work is properly cited.

Techniques based on ultrasonic guided waves (UGWs) play important roles in the structural health monitoring (SHM) of largescale civil infrastructures. In this paper, dispersion equations of longitudinal wave propagation in reinforced concrete member are investigated for the purpose of monitoring steels embedded in concrete. For a steel bar embedded in concrete, not the velocity but the attenuation dispersion curves will be affected by the concrete. The effects of steel-to-concrete shear modulus ratio, density ratio, and Poisson's ratio on propagation characteristics of guided wave in steel bar embedded in concrete were studied by the analysis of the real and imaginary parts of the wave number. The attenuation characteristics of guided waves of steel bar in different conditions including different bar concrete constraint and different diameter of steel bar are also analyzed. Studies of the influence of concrete on propagation characteristics of guided wave in steel bars embedded in concrete will increase the accuracy in judging the structure integrity and promote the level of defect detection for the steel bars embedded in concrete.

\section{Introduction}

In recent years, techniques based on ultrasonic guided waves (UGWs) have gained popularities and played important roles in the structural health monitoring (SHM) of large-scale civil infrastructures as guided waves have some important advantages, such as the capability of testing over long range with a greater sensitivity, the ability to test multilayered structures, and relatively cheapness due to simplicity and sensor cost [1]. Furthermore, frequency and mode tuning of UGWs can be utilized for evaluation of different types of deterioration or damage because UGWs have many different modes at a single frequency that are sensitive to different defects. Due to the above, various techniques based on guided wave have been proposed for damage detection and condition assessments of aerospace, mechanical, civil engineering structures, and other nondestructive test (NDT) areas. Raghavan and Cesnik [2] presented a review of guided wave for structural health monitoring. Sohn et al. [3] investigated delamination detection in composites through guided wave imaging. Song et al. $[4,5]$ developed smart piezoceramic transducers with guided wave for concrete structural health monitoring, Giurgiutiu [6] studied lamb wave generation with piezoelectric wafer active sensors for structural health monitoring (SHM). Wang et al. [7] investigated the effects of a defect's geometric parameters on the two reflection signals in pipe using guided waves and proposed a new strategy for accurate and quantitative pipeline defect characterization. Cobb et al. [8] studied the torsional guided wave attenuation in piping from coating, temperature, and large-area corrosion and obtained experimental results that wave attenuation is a good indicator of general corrosion level. Ahmad and Kundu [9] studied the influence of water flow through pipe networks on damage detection using guided waves. Beard et al. [10] used guided waves to inspect concrete reinforcing tendons and evaluated the effect of factors such as leakage and defect geometry on the inspection. Zhu et al. [11] used ultrasonic guided waves for nondestructive evaluation/structural health monitoring of trusses. The finite element method (FEM) is often carried out to assist structural design [12] and also used to study the guided waves for SHM in the civil infrastructures. Yi et al. [13] used FEM to predict the location of pitting corrosion in reinforced concrete based on guided waves. Simulation results show that it is feasible to predict corrosion monitoring based on ultrasonic guided wave in reinforced concrete. Moser et al. [14] simulated the propagation of elastic 
wave in the sheet and tubular structure using FEM. The results are fully consistent with those from experiment, which further proves the validity of the simulation in wave propagation using FEM. Chen [15] used FEM to simulate the defect monitoring by longitudinal guided wave and get the relation curves between reflection coefficient and circumferential length or axial length of the defect in pipe. He et al. [16] studied the propagation of guided waves in bending pipe using FEM. Other recent developments of guided waves for SHM were also discussed in a review prepared by Huang et al. [17].

Among the current available corrosion monitoring techniques in reinforced concrete, the technique based on ultrasonic guided wave has gained more and more popularity in the recent years due to its advantages for monitoring corrosion related damage in reinforcing bars, so it has gained popularities in the recent years [18]. Na et al. [19] used both high $(1 \mathrm{MHz})$ and low frequencies $(150 \mathrm{KHz})$ to study the effect of various bond levels by surrounding the rebar with a polyvinyl chloride polymer (PVC) pipe in RC beams and the effect of debonding location on the received waveforms. They in the same way conducted a comparison of steel concrete interfaces and glass fiber polymer-concrete interfaces using the guided waves [20,21]. Reis et al. [22] used the fundamental flexural mode below $250 \mathrm{kHz}$ for estimation of corrosion damage in steel reinforced mortar. Wu and Chang $[23,24]$ used the piezoelectric discs as sensors and actuators to detect debonding in reinforced concrete structures. A set of reinforced concrete square beam specimens with various bond levels were built and tested using guided mechanical waves at lower frequencies. He et al. [25] used frequencies between 1 and $2 \mathrm{MHz}$ to relate the effect of debonding on signals in cylindrical specimens. Dongsheng et al. [26] used five-cycle sinusoidal signals with $120 \mathrm{kHz}$ central frequency to investigate the UGW energy attenuation on the different debonding level between steel bar and concrete in both timedomain and frequency-domain analyses.

All the above test results indicated that the received waveform is less attenuated with the increase in debonding for both low and high frequencies. However, the lower frequencies showed more sensitivity to the change in bond. There was no significant change in the waveform arrival time reported. The location of debonding is not discernible through pulse transmission as reported by Evin et al. [27, 28].

These studies were mostly carried out with simulated debonding. In these cases, it is in fact the free steel bar within the concrete. There is difference in the propagation characteristics of guided waves between the free steel bars and the steel bar surrounded by concrete. However, the research on the effects of concrete on propagation characteristics of guided wave in steel bar embedded in concrete is less reported, because there is a difficulty of limitation of monitoring range of guided wave in reinforced concrete [18, 27]. Unlike guided wave propagation in other multilayered systems, such as a metal pipeline in air, wave energy in steel bars embedded in concrete, will be reduced (i.e., attenuated) at high rates due to leakage into the surrounding concrete. For the defects test of steel bar embedded in concrete, the reflected signals will be very weak, so the general time-frequency methods are difficult to identify the weak reflection signals of the defects in the detection signals.

In an infinite isotropic solid medium, only two types of independent wave propagation exist, that is, compression and shear waves. Both waves propagate with constant velocities and are nondispersive. When geometry constraints are introduced and the dimensions are close to the wavelength, the wave becomes dispersive and is called a guided wave. Longitudinal, torsional, and flexural waves propagate in isotropic cylinders. The characteristic equation for solid isotropic cylinders was originally independently derived for the special case of longitudinal propagation in the late 19th century $[29,30]$. Solutions to this equation contain the phase velocity and frequency (assuming no absorption by the medium). The derivations assume the solid cylinder is traction free and infinite in length and that the wave form has harmonic motion. The characteristic equation for torsional and flexural propagation was later derived [31,32].

In civil infrastructures, steel bars are usually embedded in concrete, so the existence of concrete is of strong interference with the integrity evaluation of steel bars, which lead to the difficult problem in the detection of steel bars using guided wave based techniques [27]. Steel bar embedded in concrete can be modeled as an isotropic solid cylinder embedded in an infinite isotropic medium. The derivation is similar to the solid cylinder in vacuum; however, displacement and stress boundary conditions must now be met at the interface between the two mediums. In the case of reinforced concrete, a solution includes phase velocity, frequency, and attenuation. So, there is obviously different propagation characteristics of UGWs in steel bars embedded in concrete compared with those in free bars. Therefore, it is important to study the influence of concrete on propagation characteristics of guided wave in steel bars embedded in concrete which will increase the accuracy in judging the structure integrity and promote the level of defect detection for the steel bars embedded in concrete $[33,34]$.

\section{Dispersion Equations of Longitudinal Guided Wave in Reinforced Concrete}

For a steel bar embedded in concrete, the guided waves propagate not only along the steel bar but also spread outward, which leads to the wave energy that diffuses from steel bar into the concrete. Furthermore, the change of wave impedance of concrete can also cause the reflection of the waves. Thus, the wave equations of guided waves in steel barconcrete system can be established based on the 3D elastic wave theory.

The simplified model of a steel bar embedded in concrete is shown in Figure 1, in which the inner layer of medium is the steel bar with the radius $a$, and peripheral medium is concrete which is infinite in radial direction. Guided waves are assumed to propagate along the $Z$-direction. In order to facilitate the analysis, the reinforcement was set as round steel bar. In the propagation in the steel bar embedded in concrete, the outward propagation and the attenuation due to 


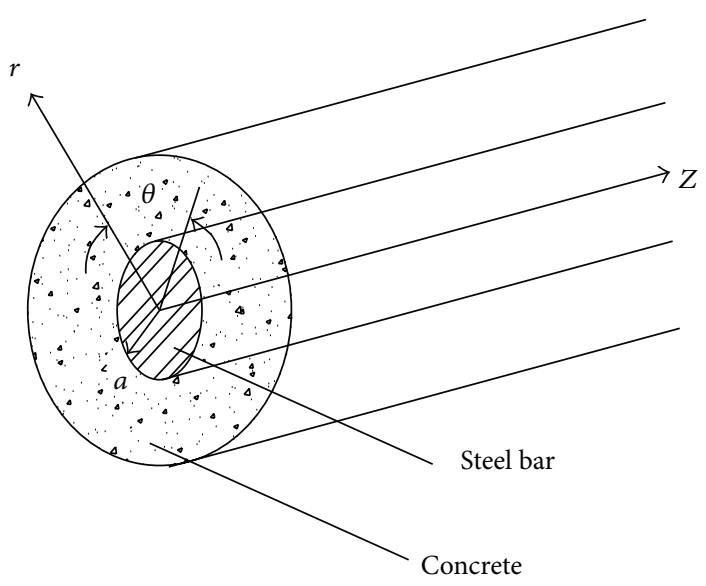

Figure 1: Simplified model of steel bar embedded in concrete.

concrete of guided waves can all be expressed by the correlation functions, in which steel bar is regarded as an isotropic elastic rod and the solution of wave equations can be solved by separation variables method.

Displacement of guided waves propagating in steel bar can be expressed by Bessel function [32]. The velocities of longitudinal and shear waves in steel bar-concrete system meet the relations: $C_{\mathrm{LS}}>C_{\mathrm{TS}}>C_{\mathrm{LC}}>C_{\mathrm{TC}}$, where $C_{\mathrm{LS}}$ and $C_{\mathrm{TS}}$ are the velocity of longitudinal and transverse waves in steel bar, respectively, and $C_{\mathrm{LC}}$ and $C_{\mathrm{TC}}$ are the velocity of longitudinal and transverse waves in concrete, respectively. Only Hankel function can meet the required condition when the wave number $k$ is a complex index [35]. Therefore, Hankel function is chosen to describe the outward propagation of waves which attenuates to zero at infinity. Based on the displacements, the stress components in both steel bar and concrete can be deduced according to the relationships of strain-displacement and stress-strain.

It is assumed that bonding condition at the interface between steel bar and concrete is good and the displacement and stress at the interface are continuous, so

$$
\left\{\begin{array}{c}
u_{r}-u_{r}^{\prime} \\
u_{\theta}-u_{\theta}^{\prime} \\
u_{z}-u_{z}^{\prime} \\
\sigma_{r r}-\sigma_{r r}^{\prime} \\
\sigma_{r \theta}-\sigma_{r \theta}^{\prime} \\
\sigma_{r z}-\sigma_{r z}^{\prime}
\end{array}\right\}=0, \quad(r=a),
$$

where $u_{r}, u_{\theta}, u_{z}$, and $u_{r}^{\prime}, u_{\theta}^{\prime}, u_{z}^{\prime}$ are the displacement components of the steel bar and concrete, respectively. $\sigma_{r r}$, $\sigma_{r \theta}, \sigma_{r z}$ and $\sigma_{r r}^{\prime}, \sigma_{r \theta}^{\prime}$, and $\sigma_{r z}^{\prime}$ are the stress components of the steel bar and concrete, respectively.
Then, substituting displacement and stress into (1), the following matrix can be obtained as follows [23]:

$$
\left\{\begin{array}{llllll}
D_{11} & D_{12} & D_{13} & D_{14} & D_{15} & D_{16} \\
D_{21} & D_{22} & D_{23} & D_{24} & D_{25} & D_{26} \\
D_{31} & D_{32} & D_{33} & D_{34} & D_{35} & D_{36} \\
D_{41} & D_{42} & D_{43} & D_{44} & D_{45} & D_{46} \\
D_{51} & D_{52} & D_{53} & D_{54} & D_{55} & D_{56} \\
D_{61} & D_{62} & D_{63} & D_{64} & D_{65} & D_{66}
\end{array}\right\}\left\{\begin{array}{c}
A_{1} \\
A_{4} \\
A_{6} \\
A_{1}^{\prime} \\
A_{4}^{\prime} \\
A_{6}^{\prime}
\end{array}\right\}=0
$$

where detailed expressions of $D_{i j}(i, j=1, \ldots, 6)$ are related to the displacement and stress components of steel bar and concrete and $A_{1}, A_{4}, A_{6}, A_{1}^{\prime}, A_{4}^{\prime}$, and $A_{6}^{\prime}$ are unknown coefficients [32].

If there is a nonzero solution to (2), the coefficient matrix of the determinant must be zero, so the following matrix equation is satisfied to obtain the general dispersion equation for the steel bar with infinite length embedded in concrete [36]:

$$
\left|D_{i j}\right|=0, \quad(i, j=1, \ldots, 6) \text {. }
$$

There are axisymmetric and nonaxisymentric modes of waves included in the propagation of guided waves in the steel bar [32]. The axisymmetric mode is the longitudinal mode $L(0, m)$, where the first index refers to the circumferential order of the mode and all of the longitudinal modes are of circumferential order 0 ; that is, $n=0$ in (3), and the second index $m$ is a counter variable which means the sequential order of the mode. Then (3) can be further simplified into two subdeterminants, after exchange of rows and columns, and can get the following equations:

$$
\underbrace{\left|\begin{array}{llll}
D_{11} & D_{12} & D_{14} & D_{15} \\
D_{31} & D_{32} & D_{34} & D_{35} \\
D_{41} & D_{42} & D_{44} & D_{45} \\
D_{61} & D_{62} & D_{64} & D_{65}
\end{array}\right|}_{D_{a}} \underbrace{\left|\begin{array}{ll}
D_{23} & D_{26} \\
D_{53} & D_{56}
\end{array}\right|}_{D_{b}}=0 .
$$


When subdeterminant $D_{a}=0$, it represents the dispersive equation of longitudinal modes, in which only nonzero displacements $\mu_{r}$ and $\mu_{z}$ are included.

When subdeterminant $D_{b}=0$, it represents the dispersive equation of torsional modes, in which only nonzero displacement $\mu_{\theta}$ is included.

Further, the following definitions are introduced [37]: $Y=$ $k a, V=\alpha_{s} a, W=\alpha_{c} a, X=\beta_{s} a$, and $U=\beta_{c} a$, where $Y$ is dimensionless wave number and $V, W, X$, and $U$ are all dimensionless. On the substitution of these definitions into the equation $D_{a}=0$, we will get the disperse equation of longitudinal mode of guided waves in reinforced concrete after simplifying the results into a simple form expressed by dimensionless quantity, as shown in the following [37]:

$$
\left.\mid \begin{array}{cc}
-V Z_{1}(V) & Y Z_{1}(X) \\
-Y Z_{0}(V) & -X Z_{0}(X) \\
\left\{-\left[\frac{\lambda_{s}}{\mu_{s}}\left(V^{2}+Y^{2}\right)+2 V^{2}\right]\right. \\
Z_{0}(V)+2 V Z_{1}(V)
\end{array}\right\} 2 Y\left[X Z_{0}(X)-Z_{1}(X)\right]
$$

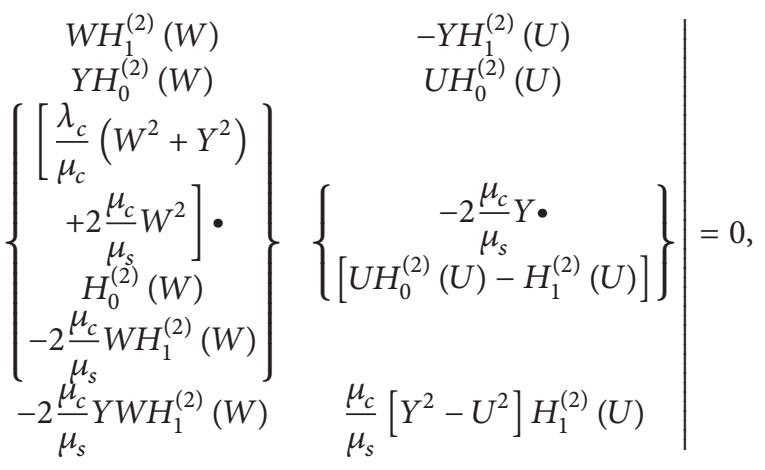

where $\mu_{s}, \rho_{s}$, and $v_{s}$ are the shear modulus, density, and Poisson's ratio of steel bar, respectively, and $\mu_{c}, \rho_{c}$, and $\nu_{c}$ are those of concrete, respectively. $Z_{0}, Z_{1}$ are Bessel functions at orders 0 and 1 , respectively. $H_{0}^{(2)}, H_{1}^{(2)}$ are Hankei functions of the second kind at orders 0 and 1 , respectively.

\section{Solution of the Dispersion Equation and the Analysis of Dispersion Curve}

The following variables are introduced: $\left(\bar{\alpha}_{s} a\right)^{2}=(\omega a)^{2} / c_{L_{s}}^{2}-$ $(k a)^{2},\left(\bar{\alpha}_{c} a\right)^{2}=(\omega a)^{2} / c_{L_{c}}^{2}-(k a)^{2},\left(\bar{\beta}_{s} a\right)^{2}=(\omega a)^{2} / c_{T_{s}}^{2}-(k a)^{2}$, and $\left(\bar{\beta}_{c} a\right)^{2}=(\omega a)^{2} / c_{T_{c}}^{2}-(k a)^{2}$. The dimensionless frequency $\Omega$ associated with angular frequency $\omega$ is defined as $\Omega=\omega a /$ $c_{L}$.

Other dimensionless quantities are given by $V^{2}=\left(c_{T_{s}} /\right.$ $\left.c_{L_{s}}\right)^{2} \cdot \Omega^{2}-Y^{2}, X^{2}=\Omega^{2}-Y^{2}, W^{2}=\left(c_{T_{s}} / c_{T_{c}}\right)^{2}\left(c_{T_{c}} / c_{L_{c}}\right)^{2} \cdot \Omega^{2}-Y^{2}$, and $U^{2}=\left(c_{T_{s}} / c_{T_{c}}\right)^{2} \cdot \Omega^{2}-Y^{2}$.

Then, the following equations can be derived from the theory of elasticity [22]:

$$
\begin{aligned}
& \frac{c_{T_{j}}}{c_{L_{j}}}=\left[\frac{1-2 v_{j}}{2\left(1-v_{j}\right)}\right]^{1 / 2}, \quad \frac{c_{T_{s}}}{c_{T_{c}}}=\left(\frac{\mu_{s}}{\mu_{c}} \times \frac{\rho_{c}}{\rho_{s}}\right)^{1 / 2}, \\
& \frac{\lambda_{j}}{\mu_{j}}=\frac{2 v_{j}}{1-2 v_{j}}, \quad \frac{\lambda_{c}}{\mu_{s}}=\frac{2 v_{c}}{1-2 v_{c}} \times \frac{\mu_{c}}{\mu_{s}} .
\end{aligned}
$$

On the substitution of these variables into (5), all variables except frequency and wave number in the dimensionless dispersion equation can be determined by the properties of steel bar and concrete which include shear modulus, Poisson's ratio, and density.

If the parameters of steel bar and concrete are known, only two unknown variables (dimensionless frequency and dimensionless wave number) are involved in (5). In this paper, the related parameters of steel bar and concrete are selected as those in Table 1.

In order to solve the relationship between dimensionless frequency and dimensionless wave numbers in the dispersion equation (5), the corresponding program is made to solve it. The solving procedures are as follows.

(1) Find the cutoff frequency of each order mode from a sweep frequency with a specified range, which is the initial root of the equation and also the initial point of the disperse curve of each order mode. Generally, there will be the same number of order modes as that of initial points which have been found within a specified range of frequency, and the corresponding number of disperse curves can be obtained.

(2) The routine starts from the initial point, in the given step and direction, and converges to the next point which is the second root of the equation.

(3) Take the second root as the initial point and repeat step 2 and so forth. The routine will end until the specified frequency or upper limit wave number is reached.

(4) Connect all the found points and a disperse curve will be achieved after.

(5) Repeat the above process from another initial point and another disperse curve will be achieved in this way. The complete solution to the equation will be gotten until all the disperse curves are finished.

Based on the solutions, the disperse curves between dimensionless frequency and dimensionless wave number of the round steel bar embedded in concrete with different material parameters will be gained by solving (5), as shown in Figures 2-4. 
TABLE 1: Related material parameters of steel bar and concrete.

\begin{tabular}{|c|c|c|c|}
\hline Parameters & $\begin{array}{l}\text { Steel } \\
\text { bar }\end{array}$ & $\begin{array}{l}\text { Ordinary } \\
\text { concrete }\end{array}$ & $\begin{array}{l}\text { High strength } \\
\text { concrete }\end{array}$ \\
\hline Poisson's ratio & 0.2865 & 0.27 & 0.20 \\
\hline Density $\left(\mathrm{kg} / \mathrm{m}^{3}\right)$ & 7932 & 2200 & 2400 \\
\hline Elastic modulus (MPa) & 210000 & 22000 & 38000 \\
\hline Shear modulus (MPa) & 81600 & 8600 & 15800 \\
\hline $\begin{array}{l}\text { Velocity of longitudinal } \\
\text { wave } C_{L}(\mathrm{~m} / \mathrm{ms})\end{array}$ & 5960 & 3540 & 4190 \\
\hline $\begin{array}{l}\text { Velocity of transverse } \\
\text { wave } C_{T}(\mathrm{~m} / \mathrm{ms})\end{array}$ & 3260 & 1980 & 2570 \\
\hline Density ratio $\rho_{s} / \rho_{c}$ & & 3.6 & 3.3 \\
\hline Shear modulus ratio $\mu_{s} / \mu_{c}$ & & 9.5 & 5.2 \\
\hline
\end{tabular}
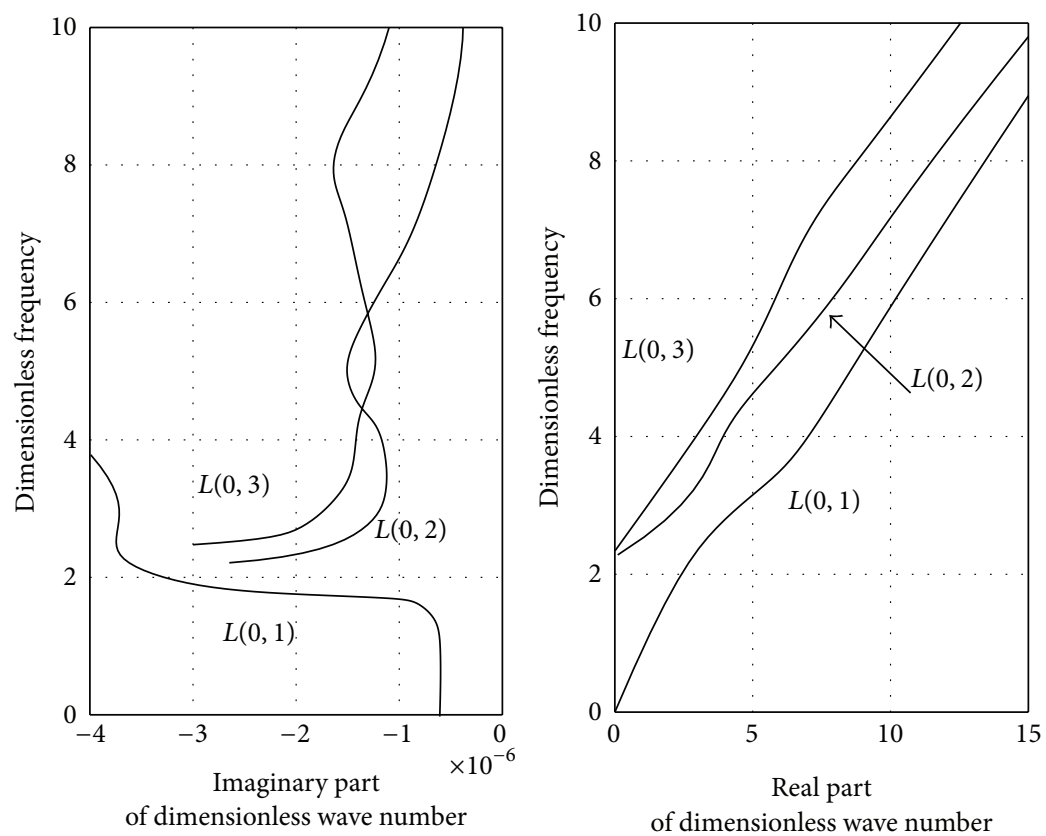

FIgURE 2: Disperse curves of the first three modes of guided waves in steel bar in the air.

From Figures 2-4, it can be observed that, for free steel bar, wave numbers are all real numbers in the solution to the disperse equation, and the corresponding modes of guided waves are free modes or modes without attenuation. For the solid round steel bar embedded in concrete, wave numbers are all complex in the solution to the disperse equation, in which the real part of wave number is corresponding to the propagation of guided waves in the steel bar and the imaginary part is corresponding to the attenuation of guided waves.

It can also be drawn from Figures 2-4 that the disperse curves between dimensionless frequency and dimensionless real wave number are almost the same whether the steel bar is in the air or embedded in concrete which means that the shear and density of surrounded concrete have no effect on the disperse curves; that is, the velocity of guided waves propagating in the steel bar is only related to the properties of steel bar.

As shown in Figures 3 and 4, the disperse curves between dimensionless frequency and dimensionless imaginary wave number change with the shear and density of concrete. When the concrete has lower shear modulus or density, the imaginary wave number is lower, the spread of the wave attenuation in steel bar is slow, and the transmission distance is further and vice versa.

From the disperse curves in Figure 3 in which steel bar is embedded in ordinary concrete, it can be seen that, for the mode $L(0,1)$, when the dimensionless frequency is less than 1.8 , the imaginary wave number is about 1.15 , which is reflecting the energy attenuation of guided waves. Analogously, from Figure 4, in which steel bar is embedded in concrete with high strength, it can be seen that, for the same 

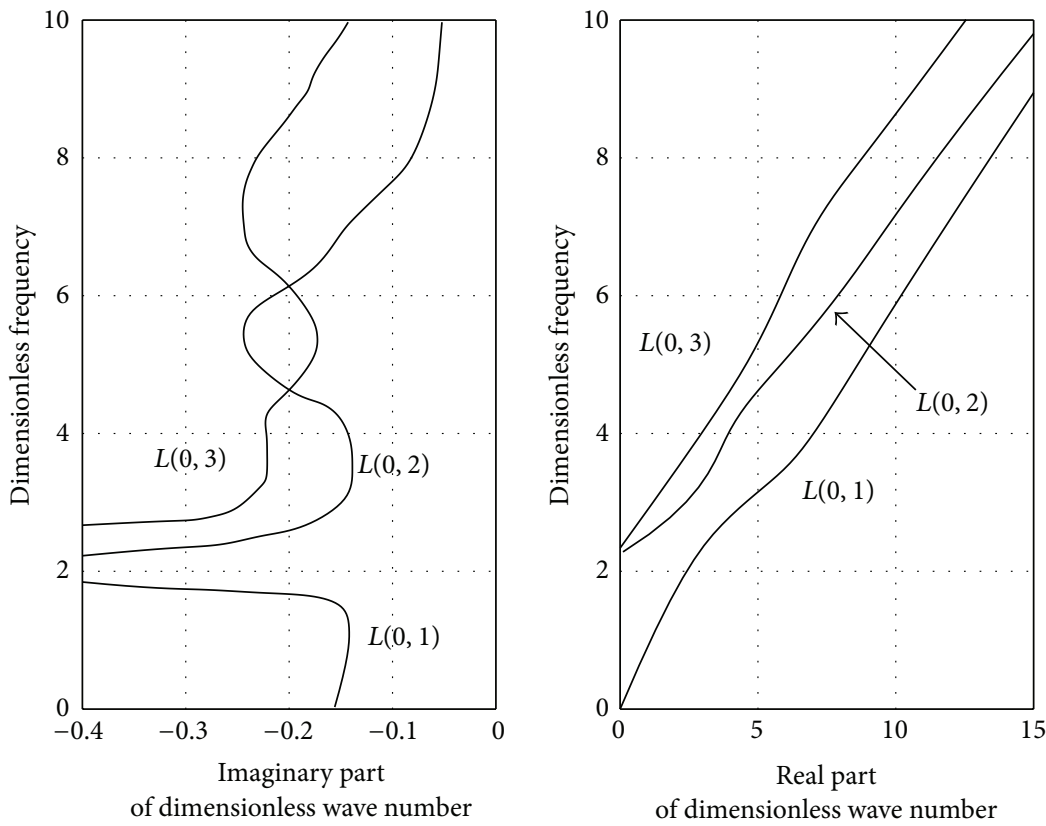

FIGURE 3: Disperse curves of the first three modes of guided waves in steel bar embedded in ordinary concrete.
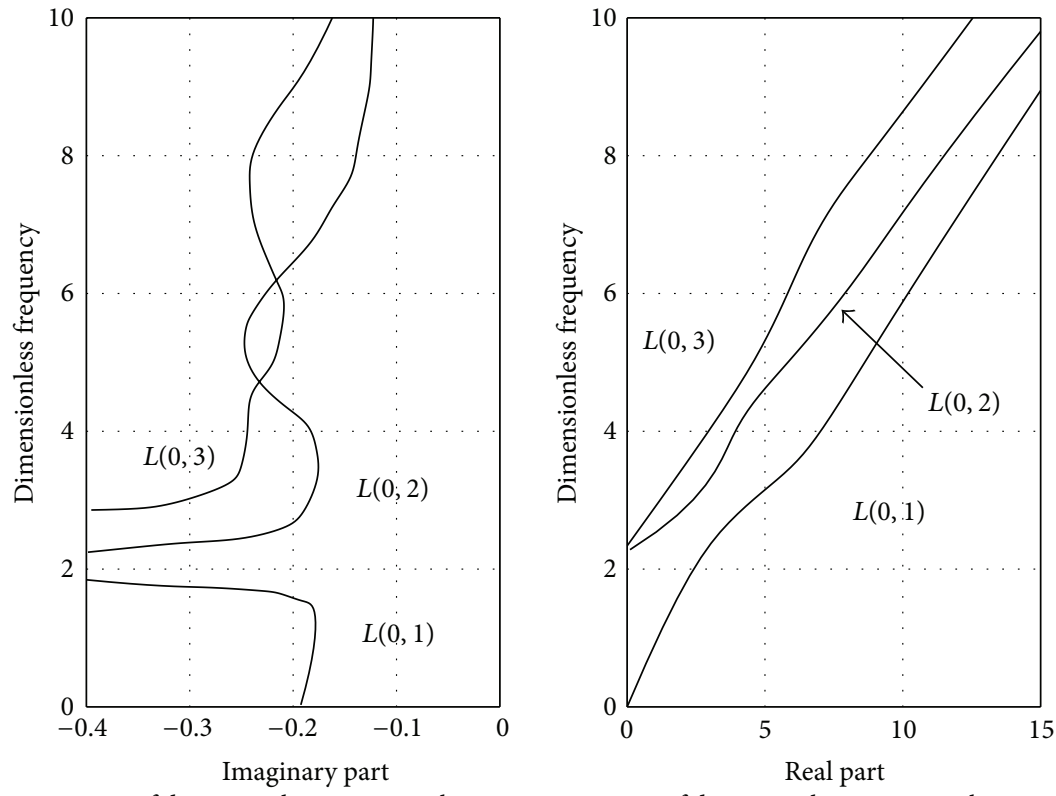

of dimensionless wave number

of dimensionless wave number

FIGURE 4: Disperse curves of the first three modes of guided waves in steel bar embedded in high strength concrete.

mode $L(0,1)$, when the dimensionless frequency is less than 1.5 , the imaginary wave number is even smaller, that is about 0.19 . This means that energy attenuation of guided waves in ordinary strength concrete is slower than that in high strength concrete. And the imaginary wave number of mode $L(0,1)$ increases quickly with the increase of frequency no matter in ordinary concrete or in high strength concrete.

\section{Parametric Analysis}

The impact of concrete on the propagation characteristics of guided wave in steel bar can be evaluated by changing the material properties of concrete, which include the factors such as steel-to-concrete shear modulus ratio, density ratio, and ratio of Poisson's ratio. Furthermore, from previous 

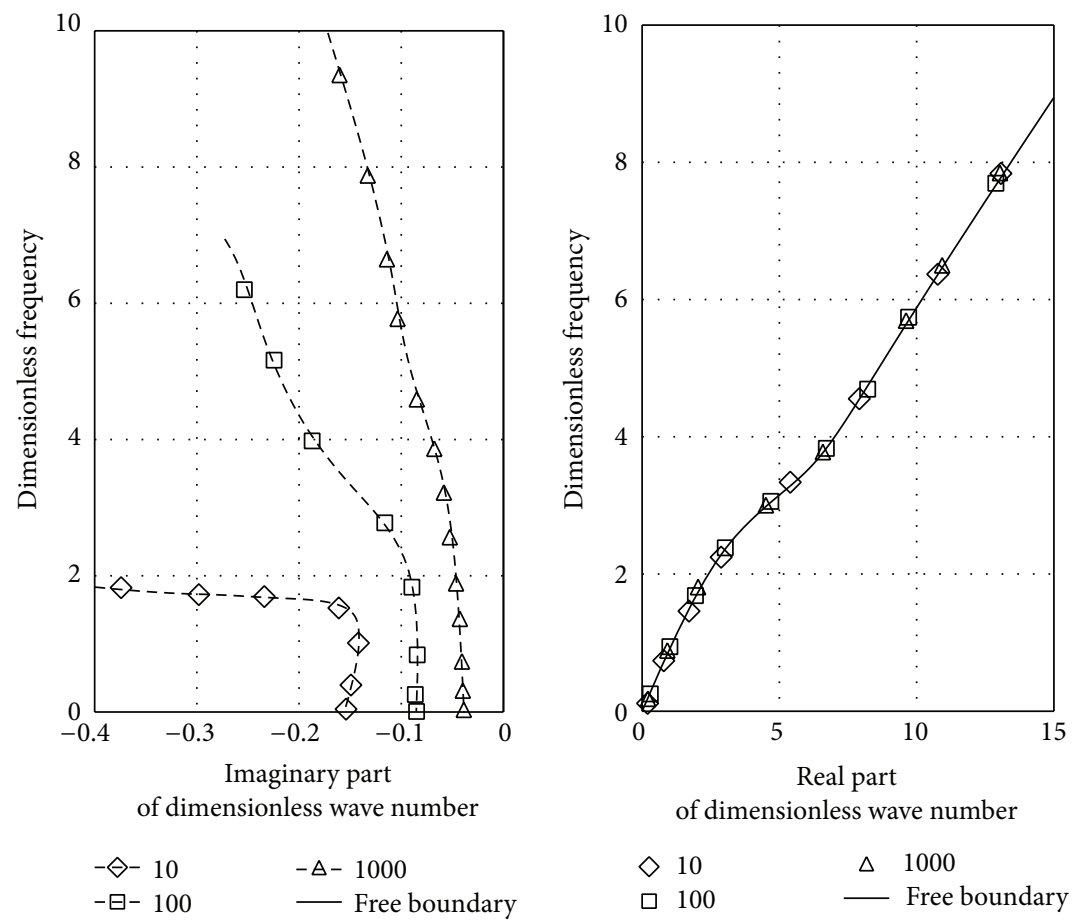

FIGURE 5: Influence on the real and imaginary parts of $L(0,1)$ by the steel-bar-to-concrete shear modulus ratio.

studies by the authors [38], it is known that the cutoff frequency of the lowest order mode $L(0,1)$ in steel bar is zero, so those modes propagating along the steel bar will always include it. Thus, the effects of related factors on the lowest order mode $L(0,1)$ are mainly taken into consideration, which are studied, respectively, in the following part.

4.1. Effect of Shear Modulus Ratio. When the steel bar is embedded in ordinary concrete, the shear modulus ratio is about 10, which will increase when the surrounding concrete becomes softer. Until it is up to a certain extent, the boundary of steel bar can be regarded as a free boundary. By changing the ratio, the relationship between dimensionless wave number and dimensionless frequency can be gained as shown in Figure 5.

From Figure 5, it can be seen that the change of shear modulus ratio has little effect on the real part of dimensionless wave number. However, with the increase of shear modulus ratio (that is, the surrounding concrete becomes more and more soft), the imaginary part of dimensionless wave number, which representing the attenuation of guided waves, becomes smaller and smaller. This shows that the attenuation of energy due to leakage into the surrounding concrete is getting smaller and farther distance of propagation can be achieved. It is considered that no energy leak into concrete in the condition of free boundary, so the dimensionless wave number will be real number.

4.2. Effect of Density Ratio. When the density of concrete is changed and other parameters remain constant, the disperse relation will surely be affected. While steel bar is embedded in high strength concrete, the steel-to-concrete density ratio is 3.6. Then, decreasing gradually the density of concrete, the steel-to-concrete density ratio will be increased and the change of the disperse curve between dimensionless wave number and dimensionless frequency will be gotten as shown in Figure 6. When the ratio is close to infinity, it indicates that the effect of surrounding concrete can be neglected, or it can be regarded as free boundary.

From Figure 6, it can be seen that the change of density ratio has little effect on the real part of dimensionless wave number. However, with the decrease of density ratio (i.e., the surrounding concrete becomes more and more dense), the imaginary part of dimensionless wave number representing the attenuation of guided waves becomes more and more large which shows that the attenuation of energy due to leakage into the surrounding concrete is getting larger and shorter distance of propagation can be achieved. It is also considered that no energy leak into concrete in the condition of free boundary, so, the dimensionless wave number is real number.

4.3. Effect of Ratio of Poisson's Ratio. As Poisson's ratio of steel bar and concrete can be changed and large changes in the Poisson's ratio of concrete from low grade to high grade take place, the effect on the disperse curve by Poisson's ratio can be gotten by assuming Poisson's ratio of steel bar as a constant of 0.2865 and changing that of concrete, as shown in Figure 7, where $v$ is Poisson's ratio of concrete.

From Figure 7, it can be found that the variations both in the real and imaginary parts of wave number are small with 

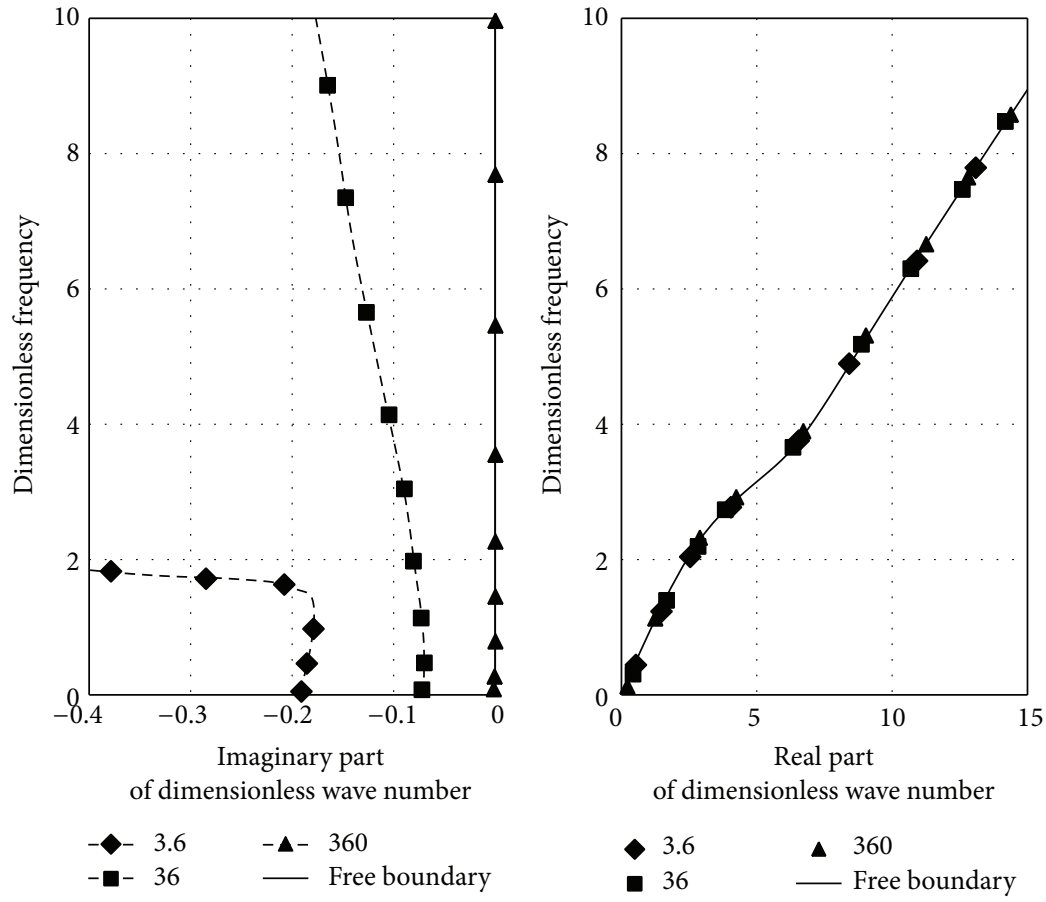

FIGURE 6: Influence on the real and imaginary parts of $L(0,1)$ by the steel-bar-to-concrete density ratio.
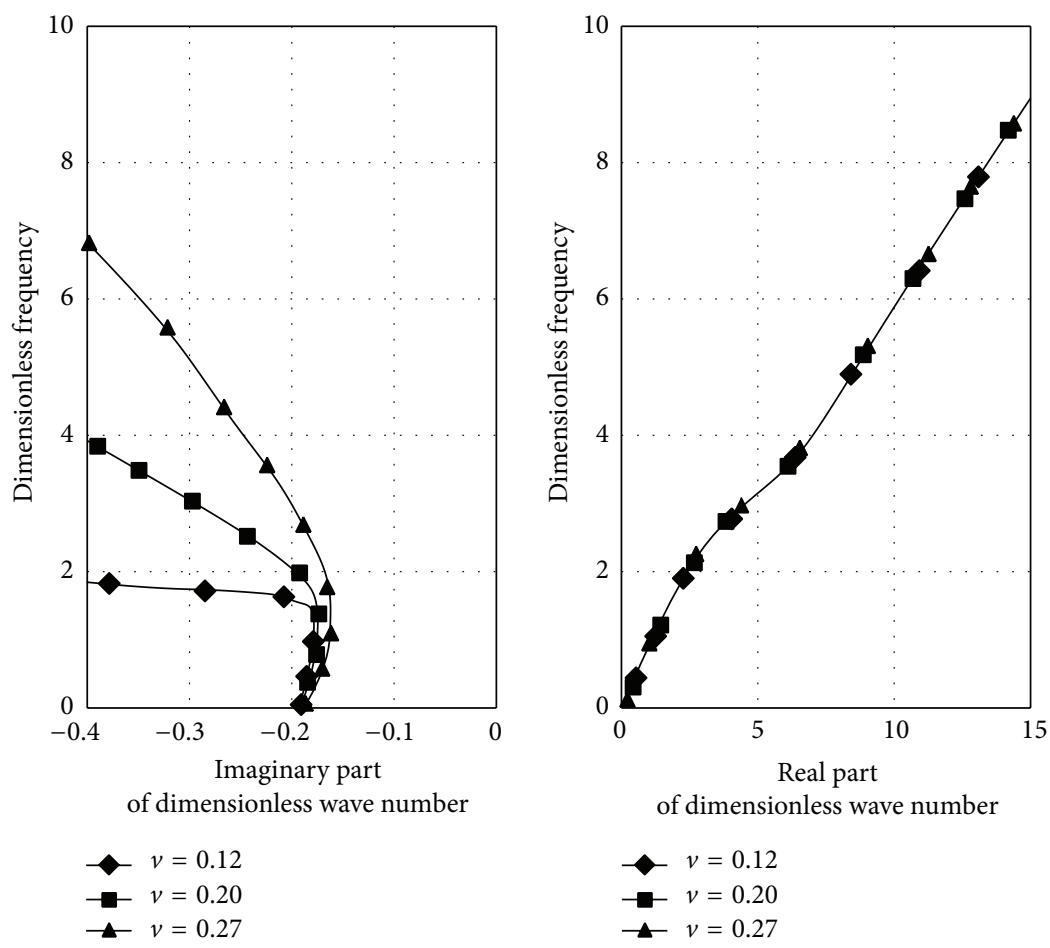

FIGURE 7: Influence on the real and imaginary parts of $L(0,1)$ by Poisson's ratio of concrete.

the change of Poisson's ratio of concrete in low frequency. When the dimensionless frequency is greater than 1.8 , the attenuation will be obviously affected with the increase of frequency and the smaller Poisson's ratio of concrete, the greater the attenuation.

\section{Attenuation Characteristics of Guided Wave in Reinforced Concrete}

Through the previous definition and analysis, it usually regarding the disperse curve between the imaginary part 
of dimensionless wave number and dimensionless frequency as the attenuation curve. In fact, the forward propagation of guided waves in steel bar is a process in which energy attenuates gradually due to the effects of constrains of concrete, impact excitation energy, material damping of steel, and defects on steel bar. If there are much residues or defects in steel bar or its characteristics is close to the surrounding concrete (close density or shear modulus), the waves will vanish due to attenuation after a short distance of propagation and then it will be very difficult to detect the defect of steel bar using the traditional echo method. Based on the research of the attenuation curve, the energy dissipation of guided waves propagating along the steel bar and the effects on the attenuation by material properties, propagation distance, and frequency can be known more clearly, which will be a benefit for the defect detection in the reinforced concrete.

When the steel bar is in the air (free steel bar), the solved imaginary wave number is very small and even close to zero because there is great difference in the physical properties between steel bar and the air. Therefore, its propagation mode is approximated free or no attenuation mode.

For the steel bar embedded in concrete, the imaginary wave number depends on the shear modulus ratio and density ratio between steel bar and concrete. The energy loss of wave can be quantified by the displacement $u$ which is expressed in the steel bar as follows [39]:

$$
u=U(a) e^{i(\omega t-k z)}=U(a) e^{i\left(\omega t-k_{r} z\right)} e^{k_{i} z},
$$

in which $k_{r}$ and $k_{i}$ are the real and imaginary parts of the wave number, respectively, $a$ is the radius of steel bar, and $z$ is the axial direction of steel bar.

Thus, the attenuation of guided wave along the steel bar can be measured using the amplitude variation of the signal along the $z$-direction. In (7), the first part $U(a)$ is the function of radius, which is a constant, taking a fixed point along the radius. The second part shows that the wave propagates along the $+Z$ direction. The third part is corresponding to the exponential increase or decrease of wave energy which depends on the symbol of $k_{i}$. The solution to the disperse equation in the steel bar surrounded by concrete shows that $k_{i}$ is negative and its displacement attenuates exponentially along the $+Z$ direction. The attenuating value of displacement along the $+Z$ direction depends on the amplitude of imaginary part of wave number. The attenuation coefficient can be signified by Neper, abbreviated as $N_{p}$,

$$
\gamma=\log _{e}\left(\frac{u_{2}}{u_{1}}\right) N_{p},
$$

where $\gamma$ is the attenuation coefficient and $u_{1}$ and $u_{2}$ are the amplitudes of signals at the initial point and last point of measuring. The ratio of amplitudes $A$ is defined as

$$
A=\frac{u_{2}}{u_{1}}=e^{\gamma} .
$$

In (9), the first two parts have no effect on the amplitude of displacement in $z$-direction and they can be substituted by a constant $E$. Thus, the attenuation coefficient is expressed as

$$
\begin{aligned}
\gamma & =\log _{e}\left(\frac{E e^{k_{i} z_{2}}}{E e^{k_{i} z_{1}}}\right)=\log _{e} e^{k_{i}\left(z_{2}-z_{1}\right)} \\
& =k_{i}\left(z_{2}-z_{1}\right) N_{p},
\end{aligned}
$$

where $z_{1}$ and $z_{2}$ are the initial and final positions of measurement. The attenuation coefficient can be turned into decibels as shown in the following:

$$
\gamma=k_{i} \text { nepers } / \mathrm{m}=8.686 k_{i} \mathrm{~dB} / \mathrm{m} .
$$

5.1. Steel Bar Embedded in Ordinary Concrete. The disperse curves for steel bar with the diameter of $22 \mathrm{~mm}$ embedded in ordinary concrete are shown in Figure 8. In the figure, the green and red dots/circles are the cutoff frequency of each mode and the roots of the dispersive equation, respectively. The involved material parameters are tabulated in Table 1.

By comparing the energy velocity curve to phase velocity curve in Figure 8, it can be seen that when the local minimum value of attenuation is achieved, the corresponding energy velocity at the frequency is the maximum. While the maximum value attenuation is reached, the corresponding energy velocity at the frequency is the minimum.

From the phase velocity disperse curves in Figure 8(a), it can be observed that the fundamental $L(0,1)$ mode is starting at zero frequency while each higher-order mode is starting from a higher cutoff frequency. Each of the higher modes shows a plateau region around the steel longitudinal bulk velocity line first and then decreases, eventually approaching the transverse wave velocity. The plateau regions correspond to the points of the maximum energy velocity (Figure $8(\mathrm{~b})$ ) and minimum attenuation (Figure $8(\mathrm{c})$ ). But $L(0,8)$ mode shows a different pattern. Instead of each plateau region belonging to a single mode, $L(0,8)$ breaks from this pattern and links the subsequent plateau regions together to form a single mode that propagates close to the longitudinal bulk velocity of steel. This $L(0,8)$ mode is chosen for study. At a frequency of $1.72 \mathrm{MHz}$, the mode exhibits global attenuation minima of $18 \mathrm{~dB} / \mathrm{m}$ and it is the fastest propagating mode. The phase velocity obtained from dispersion curve at this frequency is $6 \mathrm{Km} / \mathrm{s}$.

By (11), the corresponding amplitude ratio of signals at different propagating distance of guided waves in steel bar embedded in ordinary concrete can be calculated as tabulated in Table 2.

As can be seen from Table 2, for the steel bar embedded in ordinary concrete, after more and more energy leaks into the concrete with the increase of propagation distance, the internal energy becomes less and its particle vibration becomes more and more weak, and then the amplitude ratio of signals is becoming smaller and smaller.

Figure 9 shows the axial displacement $U_{z}$ and strain energy density (SED) distribution for $L(0,8)$ mode studied in the present investigation. The energy is concentrated in the central core portion of the bar and has relatively less surface 


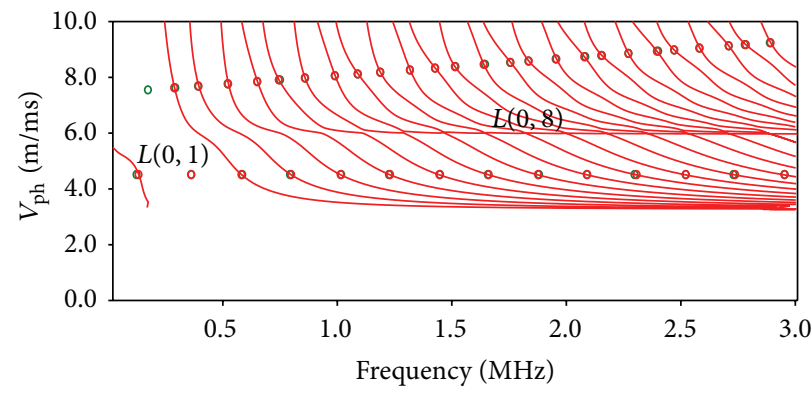

(a) Phase velocity disperse curves

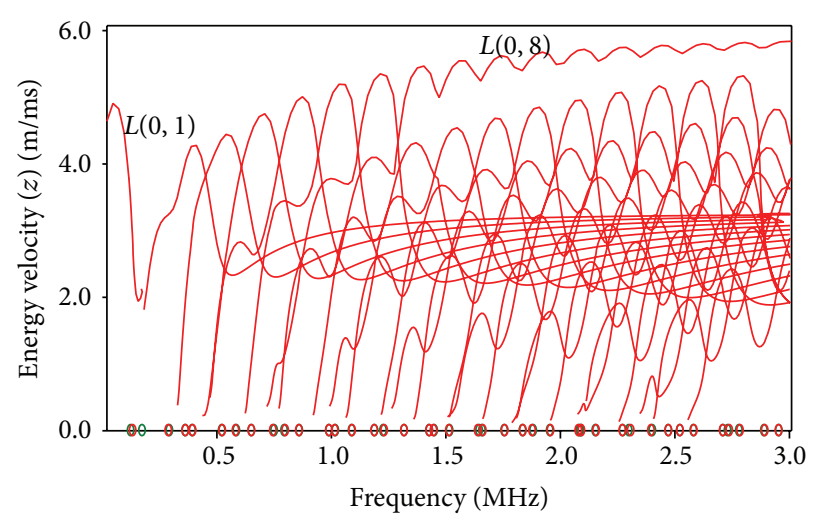

(b) Energy velocity disperse curves

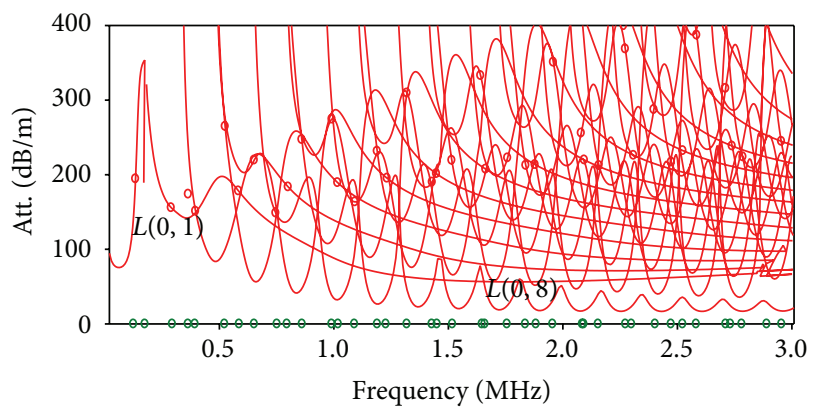

(c) Attenuation disperse curves

FIGURE 8: Disperse curves of guided waves in steel bar embedded in ordinary concrete.

TABLE 2: Attenuation coefficient and the signal amplitude ratio of mode $L(0,8)$.

\begin{tabular}{lccccccc}
\hline $\begin{array}{l}\text { Propagating } \\
\text { distance }(\mathrm{m})\end{array}$ & 0 & 1 & 1.5 & 2 & 2.5 & 3 & 5 \\
\hline $\begin{array}{l}\text { Attenuation } \\
(\mathrm{dB})\end{array}$ & 0 & -18 & -27 & -36 & -45 & -54 & -90 \\
$\begin{array}{l}\text { Amplitude } \\
\text { ratio }\end{array}$ & 1.000 & 0.1259 & 0.0450 & 0.0159 & 0.0056 & 0.0020 & 0.00003 \\
\hline
\end{tabular}

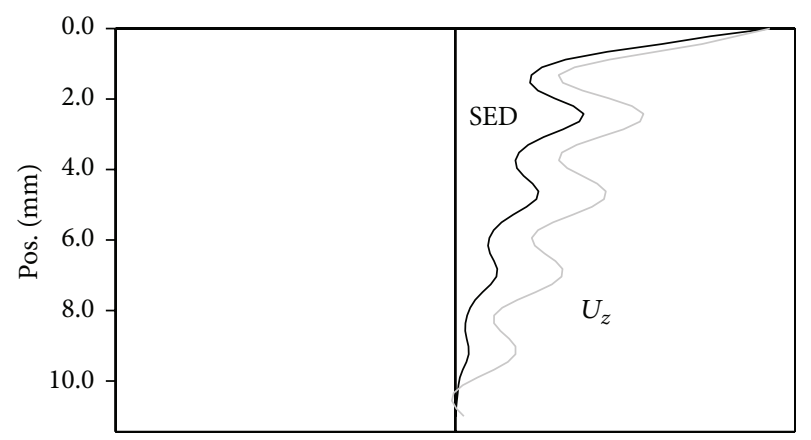

Figure 9: Mode shape of $L(0,8)$ at $f=1.72 \mathrm{MHz}$.

component. Hence, it should be more sensitive to local bar topography or loss of material changes and not the surface profile changes.
5.2. Steel Bar Embedded in Concrete with High Strength. The disperse curves for steel bar embedded in high strength concrete with the diameter of $22 \mathrm{~mm}$ are shown in Figure 10. In the figure, the green and red dots/circles are the cutoff frequency of each mode and the roots of the dispersive equation, respectively. The involved material parameters are also tabulated in Table 1.

The phase velocity disperse curves in Figure 10(a) show that, similar to those of ordinary concrete, the fundamental $L(0,1)$ mode is starting at zero frequency while each higherorder mode is starting from a higher cutoff frequency. Each of the higher modes shows a plateau region around the steel longitudinal bulk velocity line first and then decreases, eventually approaching the transverse wave velocity. The plateau regions correspond to the points of the maximum energy velocity (Figure 10(b)) and minimum attenuation (Figure $10(\mathrm{c}))$. But $L(0,14)$ mode shows a different pattern. Instead of each plateau region belonging to a single mode, $L(0,14)$ breaks from this pattern and links the subsequent plateau regions together to form a single mode that propagates close to the longitudinal bulk velocity of steel. At the frequency band corresponding to the plateau regions of modes $L(0,6)$ and $L(0,14)$, they are nearly the fastest propagating modes and exhibit global attenuation minima as shown in Figures 10(b) and 10(c). At a frequency of $1.02 \mathrm{MHz}$, the mode $L(0,6)$ has the attenuation minima of $36.8 \mathrm{~dB} / \mathrm{m}$ and at a frequency of $2.95 \mathrm{MHz}$, the mode $L(0,14)$ has the attenuation minima of $36.6 \mathrm{~dB} / \mathrm{m}$. The phase velocities of the two 


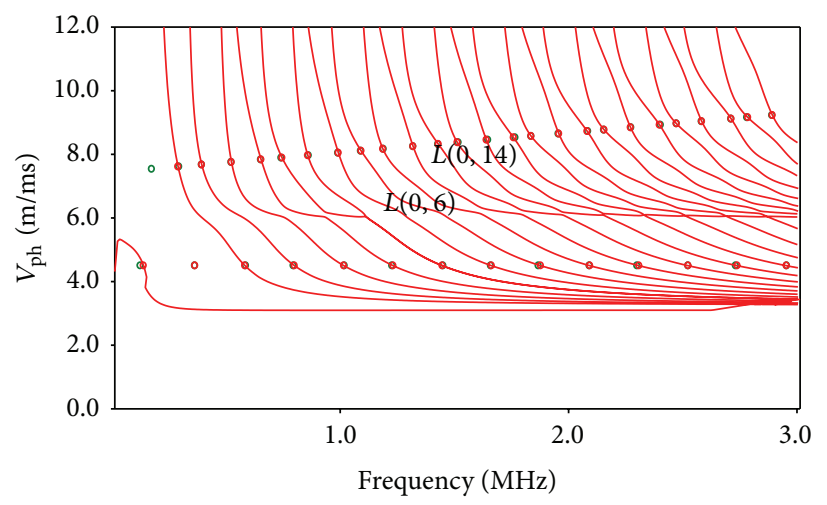

(a) Phase velocity disperse curves

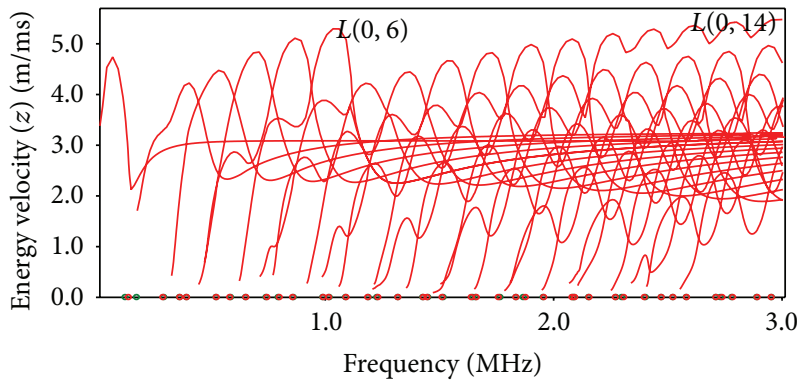

(b) Energy velocity disperse curves

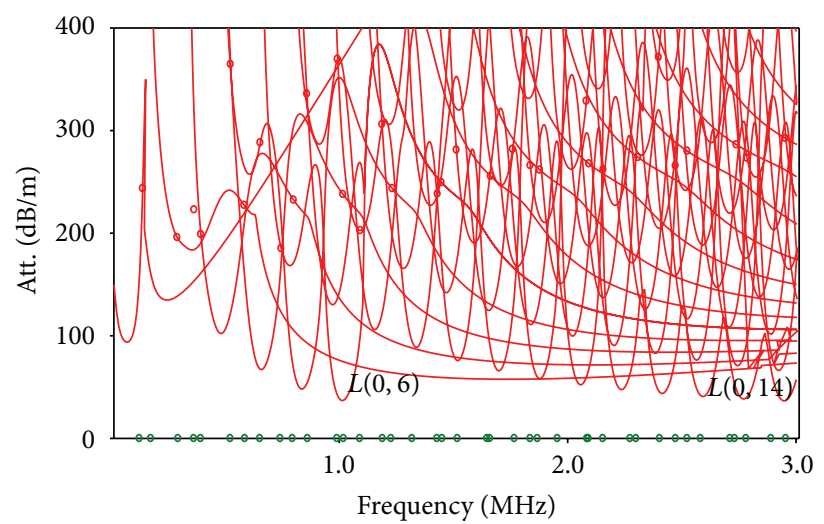

(c) Attenuation disperse curves

FIGURE 10: Disperse curves of guided waves in steel bar embedded in high strength concrete.

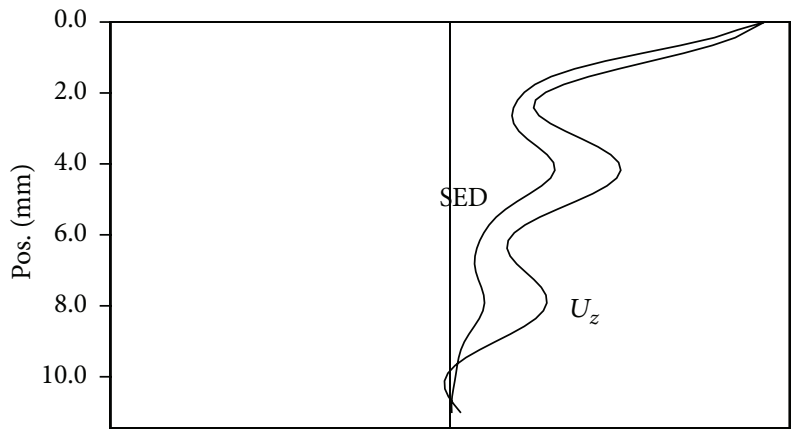

Figure 11: Mode shape of $L(0,6)$ at $f=1.02 \mathrm{MHz}$.

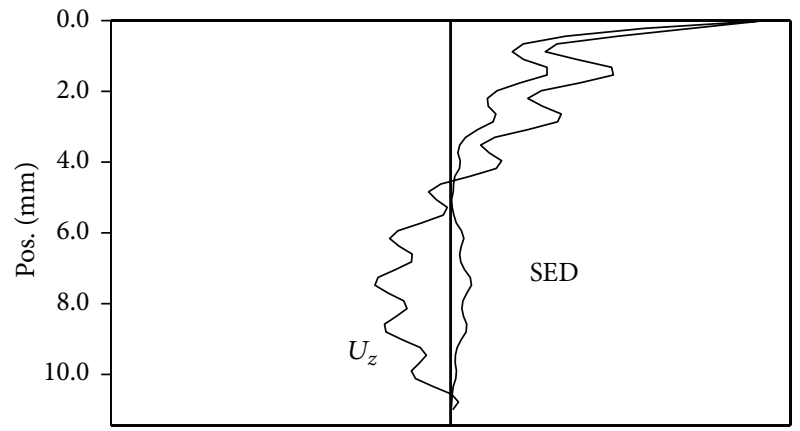

Figure 12: Mode shape of $L(0,14)$ at $f=2.94 \mathrm{MHz}$. modes as obtained from dispersion curves at the frequency corresponding to the minimum attenuation are both about $6 \mathrm{Km} / \mathrm{s}$. The attenuation value of $36.8 \mathrm{~dB} / \mathrm{m}$ is taken and then the corresponding amplitude ratio of signals at different propagating distance of guided waves in steel bar embedded in high strength concrete is calculated as tabulated in Table 3.

Figures 11 and 12 show the axial displacement $U_{z}$ and strain energy density (SED) distribution for modes $L(0,6)$ and $L(0,14)$, respectively. It can be seen that the energy of mode $L(0,6)$ is concentrated in the central core portion of the bar and has relatively less surface component. And the energy of mode $L(0,14)$ is more concentrated in the central core portion of the bar. Hence, it will be less sensitive to the surface profile changes.

From the analysis, it can be drawn that the higher the strength concrete, the more the energy leakage into concrete, the faster the attenuation of guided waves, and the smaller the amplitude ratio of signals at the same position. Guided waves at low frequency mode can propagate very far along the steel bar in the air for the little attenuation, so a few meters of steel bar can be monitored by guided waves based technique [40]. However, while the same steel bar is embedded in ordinary 
TABLE 3: Attenuation coefficient and the signal amplitude ratio of mode $L(0,6)$.

\begin{tabular}{lccccccc}
\hline Propagating distance $(\mathrm{m})$ & 0 & 1 & 1.5 & 2 & 2.5 & 3 & 5 \\
\hline Attenuation $(\mathrm{dB})$ & 0 & -36.8 & -55.2 & -73.6 & -92 & -110.4 \\
Amplitude ratio & 1.000 & 0.0150 & 0.0017 & 0.0002 & 0.00002 & $3.0 \times 10^{-6}$ & $6.2 \times 10^{-10}$ \\
\hline
\end{tabular}

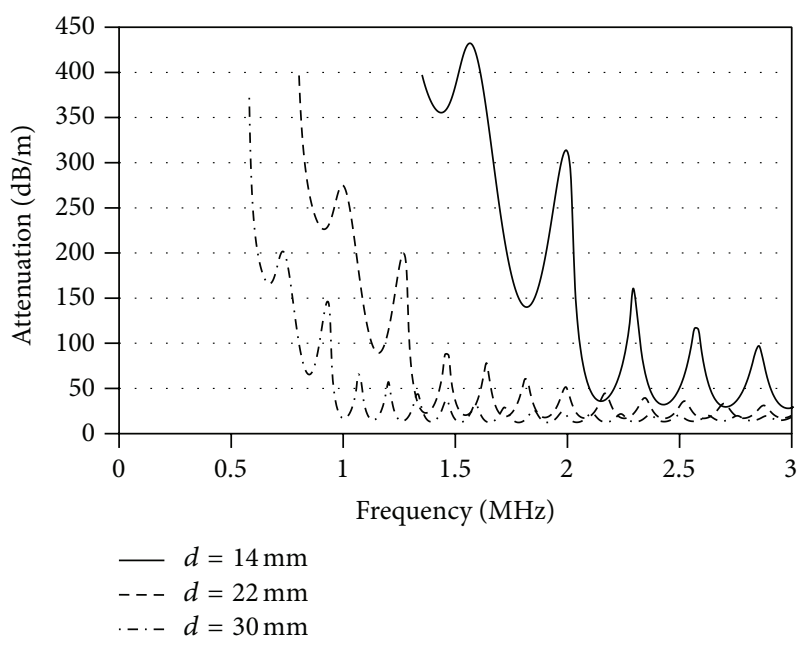

FIGURE 13: Attenuation disperse curves of mode $L(0,8)$ in steel bar with different diameter embedded in concrete.

concrete, the monitoring range is just about two meters. Even more, if it is embedded in high strength concrete, the test distance would be very limited because more and more energy leaks into the concrete and, with the increased propagation, the internal energy of steel bar becomes less and its particle vibration becomes more and more weak. Based on the above, it can be concluded that whether it is embedded in ordinary concrete or high strength concrete, the attenuation velocity of guided waves in steel bar is always high and the propagation distance is always short; that is, the detection range is very limited as the signal is very weak after two meters of propagation, which is the difficulty of nondestructive test applying guided waves in reinforced concrete.

\subsection{Influence on Attenuation of Guided Wave by Different}

Bar Diameter. For the given reinforced concrete model, the disperse curves of attenuation of longitudinal mode $L(0,8)$ for the steel bar embedded in ordinary concrete with the diameter $d=14 \mathrm{~mm}, d=22 \mathrm{~mm}$, and $d=30 \mathrm{~mm}$ are calculated, respectively, as shown in Figure 13, in which the attenuation curves are denoted by red, blue, and yellow curves, respectively, corresponding to different diameter.

As can be seen from Figure 13, the trends of attenuation disperse curves are undulate and eventually tend to be similar. At the same frequency, the value of attenuation decreases with the increase of diameter. Therefore, for the same steel bar with larger diameter embedded in the same concrete, the energy leaks less into concrete, the spread of wave energy attenuation is slower, and the spread distance is shorter.

\section{Conclusions}

In reinforced concrete structures, the propagation characteristics of guided waves in steel bar are influenced greatly by its surrounded concrete. In this paper, the impact of concrete on the propagation characteristics of guided wave in steel bar is studied by changing the material properties of concrete. It is found that the higher the excitation frequency, the more obvious the dispersion phenomenon. However, not the velocity but attenuation dispersion curves will be affected by the concrete. The shear modulus and density of concrete have no effect on the real parts of wave number; that is, the propagating velocity of guided waves in steel bar depends only on the material properties of steel bar and has nothing to do with the surrounding concrete. The imaginary part of wave number depends on the steel-bar-to-concrete shear modulus ratio and density ratio. When the concrete has lower shear modulus or density, the spread of the wave attenuation in steel bar is slow and the transmission distance is further.

For a given steel-concrete model, the attenuation extent in signal propagation can be obtained by the attenuation and frequency dispersion curve. It is found that, for the same steel bar with larger diameter embedded in the same concrete, the energy leaks less into concrete, the spread of wave energy attenuation is slower, and the spread distance is shorter. Studying the influence on propagation characteristics of guided wave in steel bars by concrete will increase the accuracy in judging the structure integrity and promote the level of defect/corrosion detection for the steel bars embedded in concrete.

\section{Conflict of Interests}

The authors declare that there is no conflict of interests regarding the publication of this paper.

\section{Acknowledgments}

This research is supported by Key Science and Technology Project from Fujian Province, China (no. 2013Y0079) and the Research Fund SLDRCE10-MB-01 from the State Key Laboratory for Disaster Reduction in Civil Engineering at Tongji University, China.

\section{References}

[1] T. R. Hay and J. L. Rose, "Interfacing guided wave ultrasound with wireless technology," in Smart Structures and MaterialsSensors and Smart Structures Technologies for Civil, Mechanical, and Aerospace Systems, vol. 5391 of Proceedings of the SPIE, pp. 314-320, San Diego, Calif, USA, March 2004. 
[2] A. Raghavan and C. E. S. Cesnik, "Review of guided-wave structural health monitoring," Shock and Vibration Digest, vol. 39, no. 2, pp. 91-114, 2007.

[3] H. Sohn, D. Dutta, J. Y. Yang et al., "Delamination detection in composites through guided wave field image processing," Composites Science and Technology, vol. 71, no. 9, pp. 1250-1256, 2011.

[4] G. B. Song, Y. L. Mo, K. Otero, and H. Gu, "Health monitoring and rehabilitation of a concrete structure using intelligent materials," Journal of Smart Materials and Structures, vol. 15, no. 2, pp. 309-314, 2006.

[5] R. L. Wang, H. Gu, Y. L. Mo, and G. Song, "Proof-of-concept experimental study of damage detection of concrete piles using embedded piezoceramic transducers," Smart Materials and Structures, vol. 22, no. 4, Article ID 042001, 2013.

[6] V. Giurgiutiu, "Lamb wave generation with piezoelectric wafer active sensors for structural health monitoring," in Smart Structures and Materials 2003: Smart Structures and Integrated Systems, vol. 5056 of Proceedings of SPIE, San Diego, Calif, USA, March 2002.

[7] X. Wang, P. W. Tse, C. K. Mechefske, and M. Hua, "Experimental investigation of reflection in guided wave-based inspection for the characterization of pipeline defects," NDT \& E International, vol. 43, no. 4, pp. 365-374, 2010.

[8] A. C. Cobb, H. Kwun, L. Caseres, and G. Janega, "Torsional guided wave attenuation in piping from coating, temperature, and large-area corrosion," NDT and E International, vol. 47, pp. 163-170, 2012.

[9] R. Ahmad and T. Kundu, "Influence of water flow through pipe networks for damage detection using guided waves," in Nondestructive Testing of Materials and Structures, vol. 6 of RILEM Bookseries, pp. 681-687, 2013.

[10] M. D. Beard, M. J. S. Lowe, and P. Cawley, "Inspection of steel tendons in concrete using guided waves," Review of Quantitative Nondestructive Evaluation, vol. 22, pp. 1139-1147, 2003.

[11] X. P. Zhu, P. Rizzo, A. Marzani, and J. Bruck, "Ultrasonic guided waves for nondestructive evaluation/structural health monitoring of trusses," Journal of Measurement Science and Technology, vol. 21, no. 4, Article ID 045701, 2010.

[12] Z. P. Zheng, Y. Lei, and X. Xue, "Numerical simulation of monitoring corrosion in reinforced concrete based on ultrasonic guided waves," The Scientific World Journal, vol. 2014, Article ID 752494, 9 pages, 2014.

[13] T. Yi, H. Li, and M. Gu, "Recent research and applications of GPS-based monitoring technology for high-rise structures," Structural Control and Health Monitoring, vol. 20, no. 5, pp. 649670, 2013.

[14] F. Moser, L. J. Jacobs, and J. Qu, "Modeling elastic wave propagation in waveguides with the finite element method," Journal of NDT \& E International, vol. 32, no. 4, pp. 225-234, 1999.

[15] Z. B. Cheng, Numerical simulation and experimental investigation on crack detection in pipes using ultrasonic guided waves [M. E. thesis], Taiyuan University of Technology, 2004.

[16] C. H. He, Y. X. Sun, Z. Liu, X. Wang, and B. Wu, "Finite element analysis of defect detection in curved pipes using ultrasonic guided waves," Journal of Beijing University of Technology, vol. 32, no. 4, pp. 289-294, 2006 (Chinese).

[17] G. L. Huang, F. Song, and X. Wang, "Quantitative modeling of coupled piezo-elastodynamic behavior of piezoelectric actuators bonded to an elastic medium for structural health monitoring: a review," Sensors, vol. 10, no. 4, pp. 3681-3702, 2010.
[18] Y. Lei and Z. P. Zheng, "Review of physical based monitoring techniques for condition assessment of corrosion in reinforced concrete," Mathematical Problems in Engineering, vol. 2013, Article ID 953930, 14 pages, 2013.

[19] W. Na, T. Kundu, and M. R. Ehsani, "Ultrasonic guided waves for steel bar concrete interface testing," Journal of Material Evaluation, vol. 60, no. 3, pp. 437-444, 2002.

[20] W. Na, T. Kundu, and M. R. Ehsani, "Lamb waves for detecting delamination between steel bars and concrete," Computer-Aided Civil and Infrastructure Engineering, vol. 18, no. 1, pp. 58-63, 2003.

[21] W. Na, T. Kundu, and M. R. Ehsani, "A comparison of steel/concrete and glass fiber reinforced polymers/concrete interface testing by guided waves," Journal of Material Evaluation, vol. 61, no. 2, pp. 155-161, 2003.

[22] H. Reis, B. L. Ervin, D. A. Kuchma, and J. T. Bernhard, "Estimation of corrosion damage in steel reinforced mortar using guided waves," Journal of Pressure Vessel Technology, vol. 127, no. 3, pp. 255-261, 2005.

[23] F. Wu and F. Chang, "Debond detection using embedded piezoelectric elements in reinforced concrete structures, part I: experiment," Structural Health Monitoring, vol. 5, no. 1, pp. 5-15, 2006.

[24] F. Wu and F.-K. Chang, "Debond detection using embedded piezoelectric elements for reinforced concrete structures-part II: analysis and algorithm," Structural Health Monitoring, vol. 5, no. 1, pp. 17-28, 2006.

[25] C. He, J. K. van Velsor, C. M. Lee, and J. L. Rose, "Health monitoring of rock bolts using ultrasonic guided waves, quantitative nondestructive evaluation," in Proceedings of the AIP Conference, pp. 195-201, AIP, Reston, Va, USA, 2006.

[26] L. Dongsheng, R. Tao, and Y. Junhui, "Inspection of reinforced concrete interface delamination using ultrasonic guided wave non-destructive test technique," Science China Technological Sciences, vol. 55, no. 10, pp. 2893-2901, 2012.

[27] B. L. Ervin and H. Reis, "Longitudinal guided waves for monitoring corrosion in reinforced mortar," Journal of Measurement Science and Technology, vol. 19, no. 5, Article ID 055702, 2008.

[28] B. L. Ervin, D. A. Kuchma, J. T. Bernhard, and H. Reis, "Monitoring corrosion of rebar embedded in mortar using high-frequency guided ultrasonic waves," Journal of Engineering Mechanics, vol. 135, no. 1, pp. 9-19, 2009.

[29] L. Pochhammer, "Uber die fortpflanzungsgeschwindigkeiten kleiner schwingungen in einem unbegrenzten isotropen kreiszylinder," Journal für die Reine und Angewandte Mathematik, vol. 81, pp. 324-336, 1876.

[30] C. Chree, "The equations of an isotropic elastic solid in polar and cylindrical coordinates, their solutions and applications," Transactions of the Cambridge Philosophical Society, vol. 14, pp. 250-369, 1889.

[31] D. Bancroft, "The velocity of longitudinal waves in cylindrical bars," Physical Review, vol. 59, no. 7, pp. 588-593, 1941.

[32] J. L. Rose, Ultrasonic Waves in Solid Media, Cambridge University Press, Cambridge, UK, 1999.

[33] T. H. Yi, H. N. Li, and H. M. Sun, "Multi-stage structural damage diagnosis method based on "energy-damage" theory," Smart Structures and Systems, vol. 12, no. 3-4, pp. 345-361, 2013.

[34] T. H. Yi and H. N. Li, "Methodology developments in sensor placement for health monitoring of civil infrastructures," 
International Journal of Distributed Sensor Networks, vol. 2012, Article ID 612726, 11 pages, 2012.

[35] A. A. Hanifah, A theoretical evaluation of guided waves in deep foundations [Dissertation], Northwestern University, Evanston, Ill, USA, 1999.

[36] B. N. Pavlakovic and M. Lowe, Disperse Users Manual, Version 2.0.16B, University of London, 2003.

[37] R. N. Thurston, "Elastic waves in rods and clad rods," Journal of the Acoustical Society of America, vol. 64, no. 1, pp. 1-37, 1978.

[38] Z. P. Zheng, Y. Lei, X. P. Cui, and Y. Song, "Non-destructive test of the steel bar by using piezoceramics sheets," in Proceeds of $3 \mathrm{rd}$ International Conference on Advanced Measurement and Test, Xiamen, China, 2013.

[39] D. Liu, A. Zhou, and Y. Liu, "Torsional wave in finite length intact pile," Chinese Journal of Applied Mechanics, vol. 2, no. 2, pp. 258-263, 2005.

[40] J. L. Rose, "A baseline and vision of ultrasonic guided wave inspection potential," Journal of Pressure Vessel Technology, Transactions of the ASME, vol. 124, no. 3, pp. 273-282, 2002. 

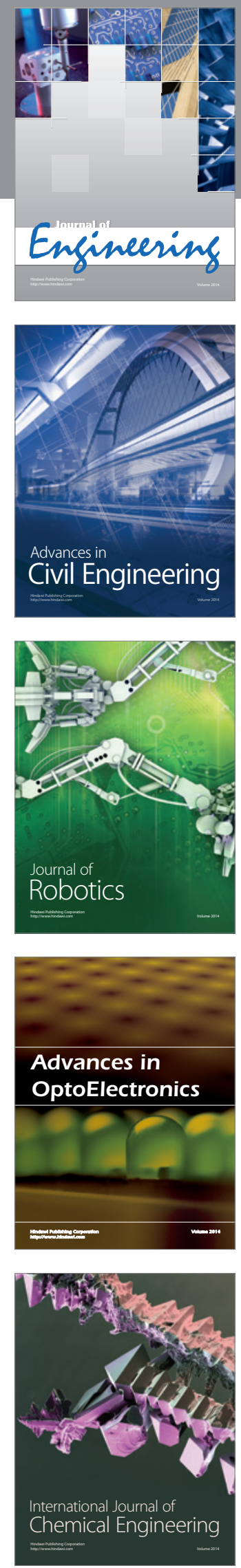

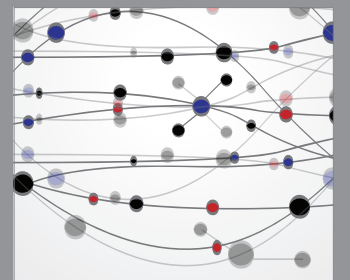

The Scientific World Journal
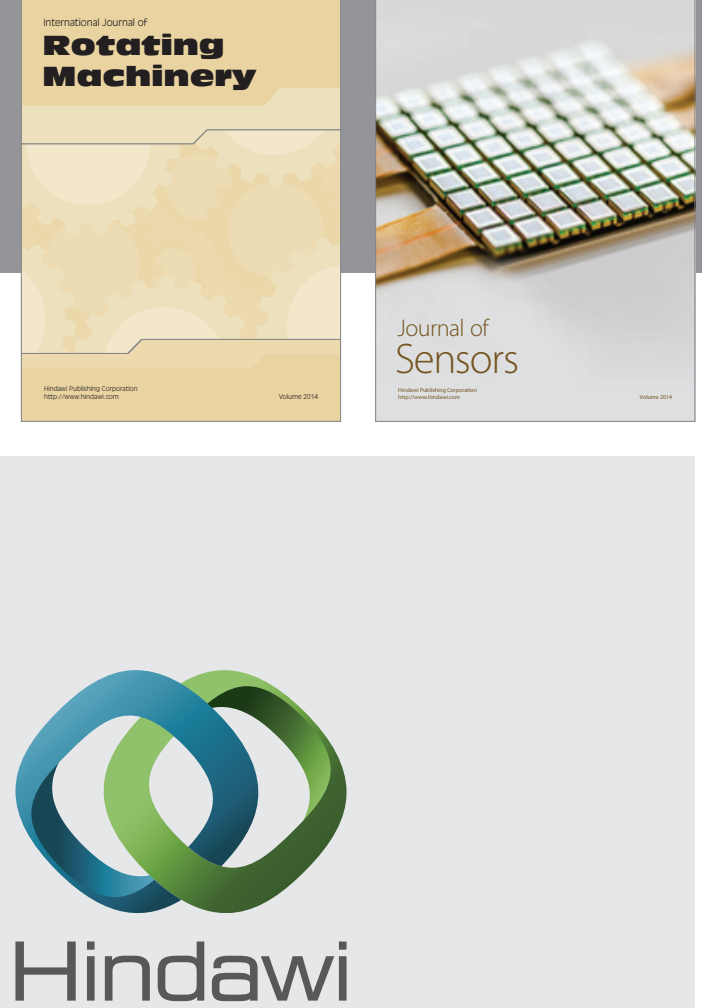

Submit your manuscripts at http://www.hindawi.com
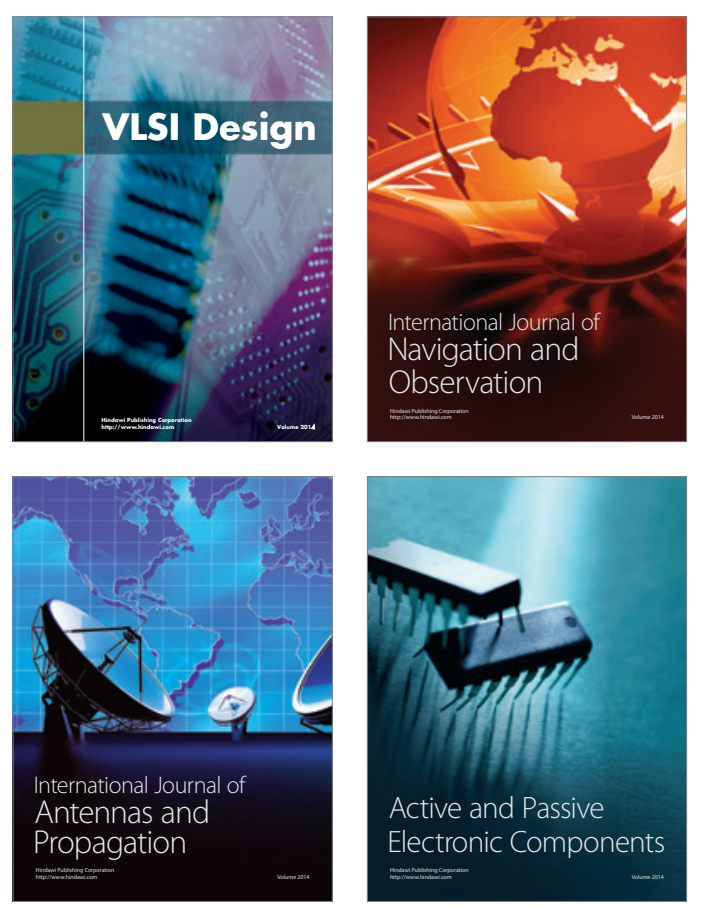
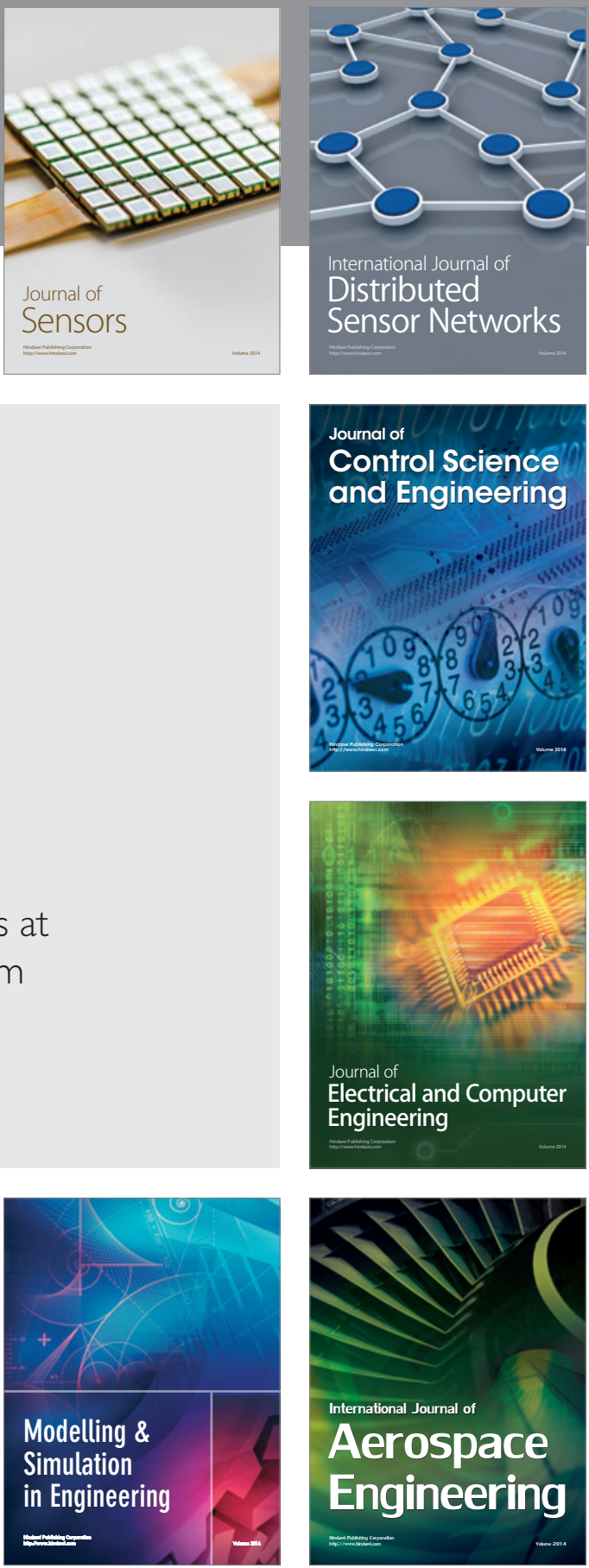

Journal of

Control Science

and Engineering
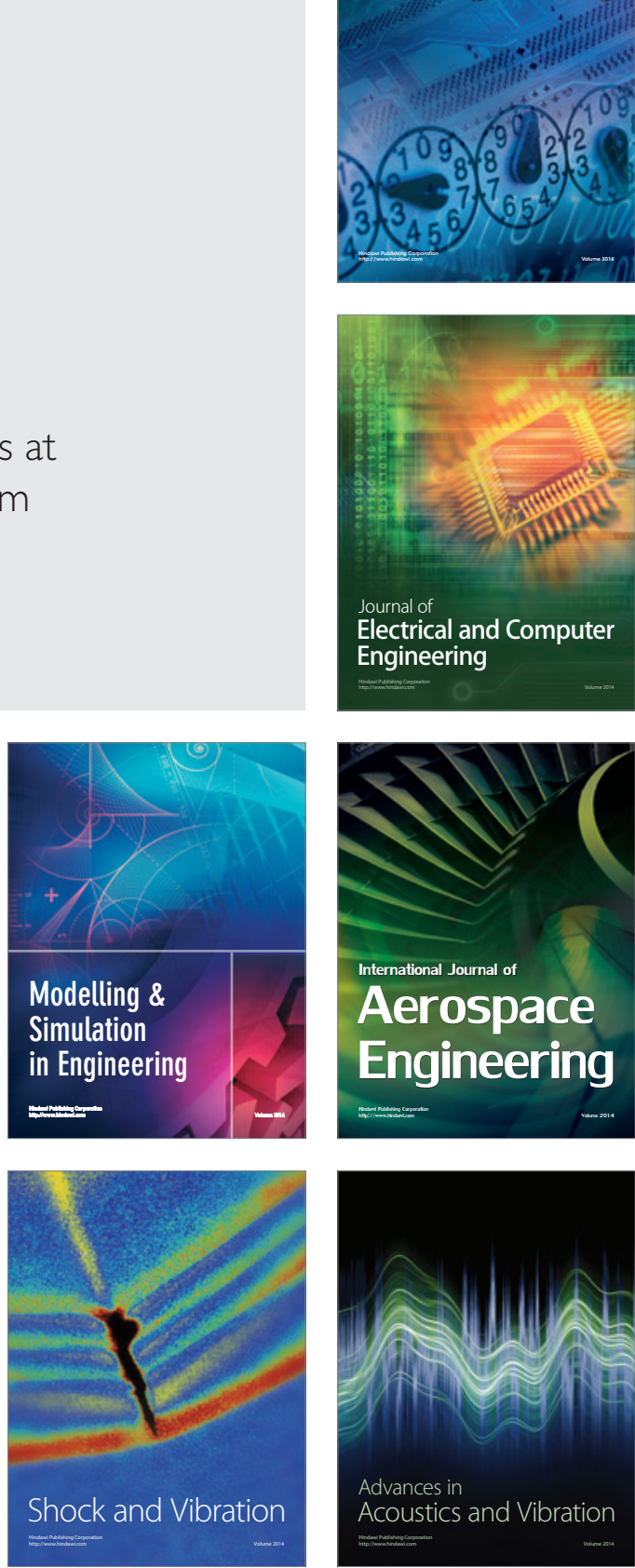